\title{
The Swedish modification of the tracheostomy tube to permit speech*
}

\author{
G Andersson MD
}

Consultant in Anaesthesia and Intensive Care, Danderyd Hospital, 18288 Danderyd, Sweden.

Over the last 20 years the Respiratory Unit at Danderyd Hospital, Sweden has treated and rehabilitated more than 90 patients on domiciliary ventilation, the majority of whom require night-time ventilation. Sixteen patients (11 men and 5 women) with high spinal cord lesions have been treated. Of these 16 patients, 9 are using diaphragmatic pacing. Every patient has a customised tube. If the patient can use diaphragmatic pacing the tube, as a rule, is single fenestrated. In patients using the ventilator full time, the tube is made to fit snugly in the trachea. When such a tube fits 'as a bullet in its tube' there is no need for a cuff. The patient has adequate ventilation and the small leak around the tube also permits good speech function. Sometimes a PEEP-valve is used to get a better voice.

\section{Keywords: tracheostomy tube; speech; domiciliary ventilation; diaphragmatic ventilation; spinal cord injury.}

\section{Methods}

For the tube material we use silver, silicone or silicone rubber, sometimes combining the two latter. The patient has a fibreoptic tracheoscopy in order to make a tube of appropriate curvature. When the custommade tube is produced the tracheal stoma may need to be refashioned to accommodate the optimal outer diameter of the tube (Fig 1). For good speech, when the patient is connected to the ventilator, it is of vital importance to have a good tube fit in the stoma.

\section{Tracheostomy management and tube selection}

In adults I prefer an oval incision in the anterior wall of the trachea, midway between the cricoid cartilage and the sternal notch. This oval incision avoids the risk of an inturned flap which could contribute to tracheal stenosis. The technique for adults with an oval incision comes from a well known thoracic surgeon in Sweden (Eric

\footnotetext{
*Paper read at the first European Conference on Domiciliary Ventilation and High Spinal Cord Lesion, Southport, England, October 1991.
}

Carlens). The tailor-made tube system is ideally constructed during the first week following the tracheostomy. If a longer period of time has elapsed a plastic operation on the tracheostomy is often necessary to be able to work on the permanent tube system. Normally this can be performed under local anaesthesia. In most cases an incision has to be made in the midline downwards from the skin to the anterior wall to the trachea and often includes division of cartilage in the anterior wall. Old scar tissue in the edges of the incision must be excised. After refashioning the stoma I test it with a single fenestrated tube, silver or silicone, of appropriate dimension, and the tip of the tube is checked endoscopically. It is important to find the right curvature of the tube to avoid pressure effects on the tracheal walls (Fig 2). Furthermore, there must be an adequate distance from the main bronchi so that suction to both lungs can be easily performed.

Patients from other regions of Sweden may initially use a prototype of the tube. Subsequently a copy can be made at our unit and the patient will get the new permanent system without delay.

Direct vision of the position of the fenestration of the test tube provides an easy 


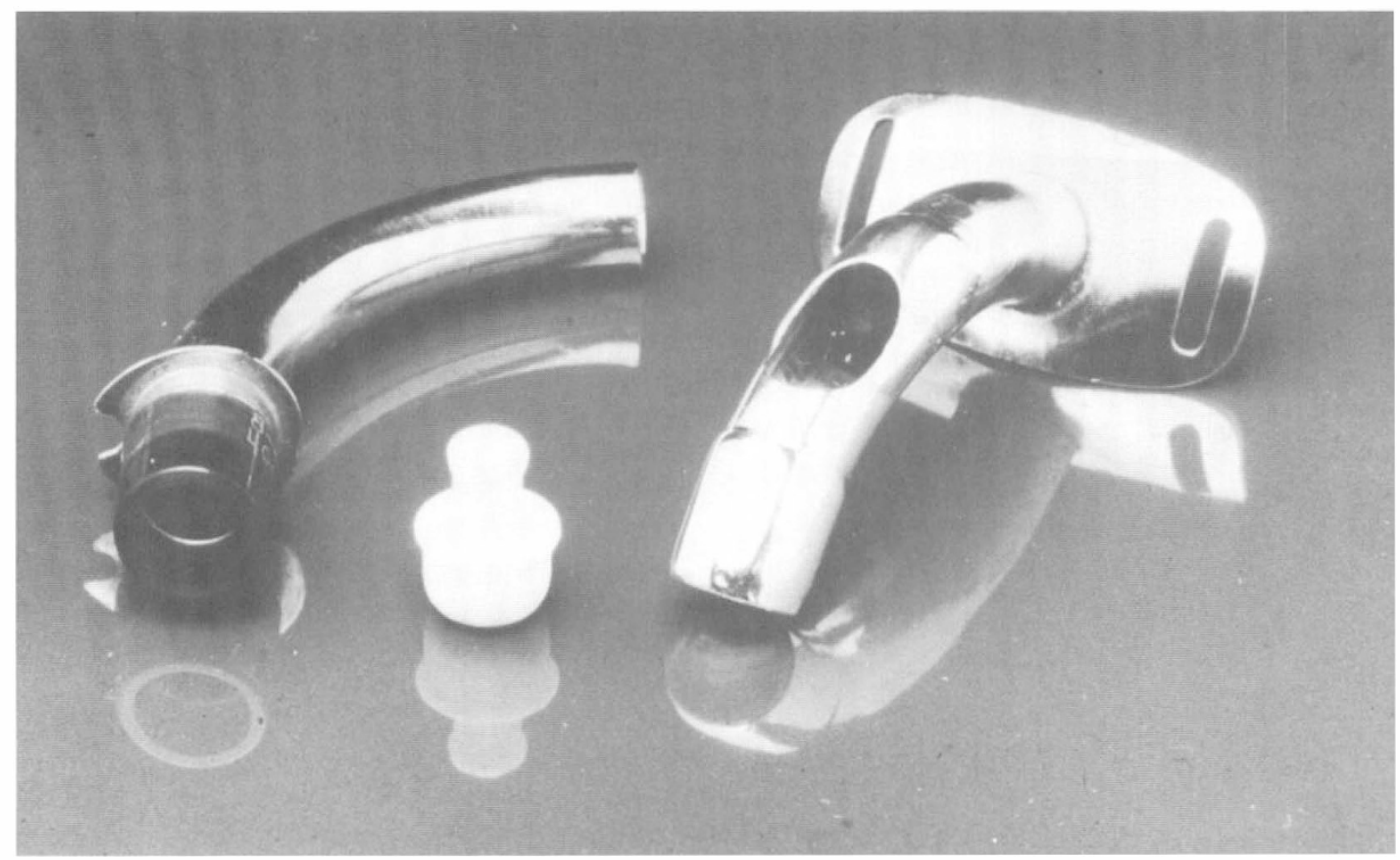

Figure 1 A short silver inner tube with a full length outer single fenestrated silicone tube fitting tightly in the tracheostoma and in the trachea.

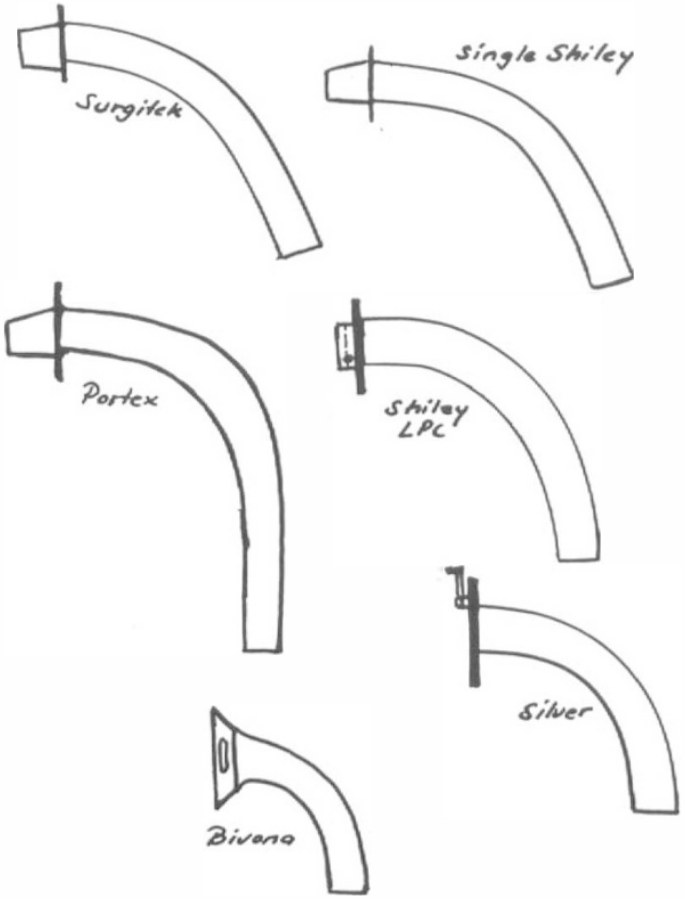

Figure 2 Curvatures of different tracheostomy tubes. method to tailor-make the fenestration of the permanent system. Granulation tissue, if present in the front wall of the trachea can be easily scraped out through the fenestrated tube with a curette. The granulations are curetted out against the silver edge of the fenestration. The insufflation leak is estimated when the patient is connected to the ventilator via the tube being assessed. In this way one can gradually work out the exact tube dimension and the position of the fenestration. Sometimes the tube dimension as such is adequate for ventilation. If the tube fits snugly in the trachea 'as a bullet in its tube' there is no need for a cuff. The patient obtains adequate ventilation and the small leak permits speech function as well. Sometimes there is a need to construct silver cuffs and sometimes the length of the standard tube must be altered. In other cases we use silicone tubes or silicone rubber with a special soft high volume cuff. If needed we alter the length of the standard tube. The tube should fit snugly both in the stoma and in the trachea. This means there should be no air leak in the stoma but a 
sufficient leak around the tube to permit speech function. Sometimes we even use PEEP-valve to improve the latter.

An inner tube covering the fenestration is needed when the ventilator is connected or if manual ventilation with a bag is used. Single fenestrated tubes are used in patients who are using diaphragmatic pacing in the day or during both day and night, but the possibility of needing to be connected to a ventilator is always considered. Patients who need ventilation both during the day and at night have tailor-made tubes with fenestrations. In some patients using diaphragmatic pacing there is impairment of vocal cord function and they cannot plug the fenestrated tube. They need to use a speaking flap valve.

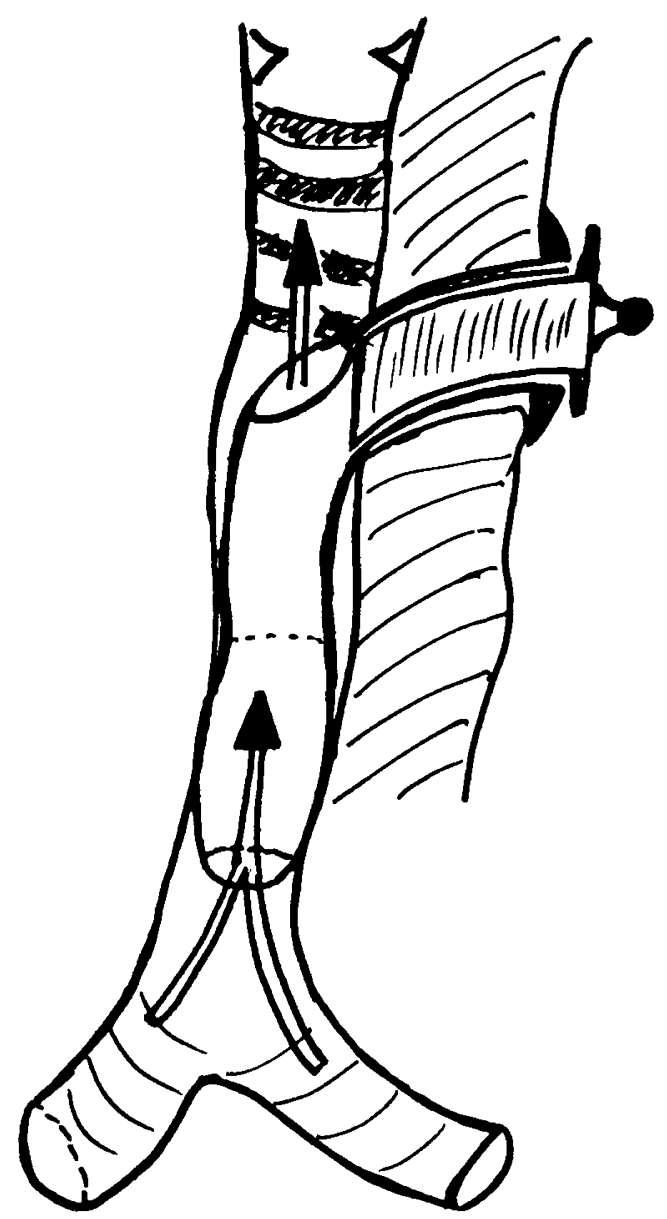

Figure 3 A silver fenestrated tube.
The speaking flap valve

Using a fenestrated tube, inspiration takes place with a low resistance and during expiration the air stream is directed the normal way thus permitting speech and a better control of the swallowing process, (Fig 3). The speaking flap valve consists of a filter-house and a flap valve. The airstream is guided by a thin silicone membrane in the cover of the filter-housing and has a central fixation. Being almost silent this product improves the quality for daily living. Added oxygen may be given through the filterhousing. By removing the cap the filter can be easily changed every second or third day. Whenever possible a fenestrated tube should be used (Fig 4).

\section{Conclusion and longterm management}

The high quality tube material makes it possible to use the same custom-made tubes for longer periods. Usually two permanent tube systems are made. In this way they can be regularly changed and disinfected and, being used many times over, they are cost

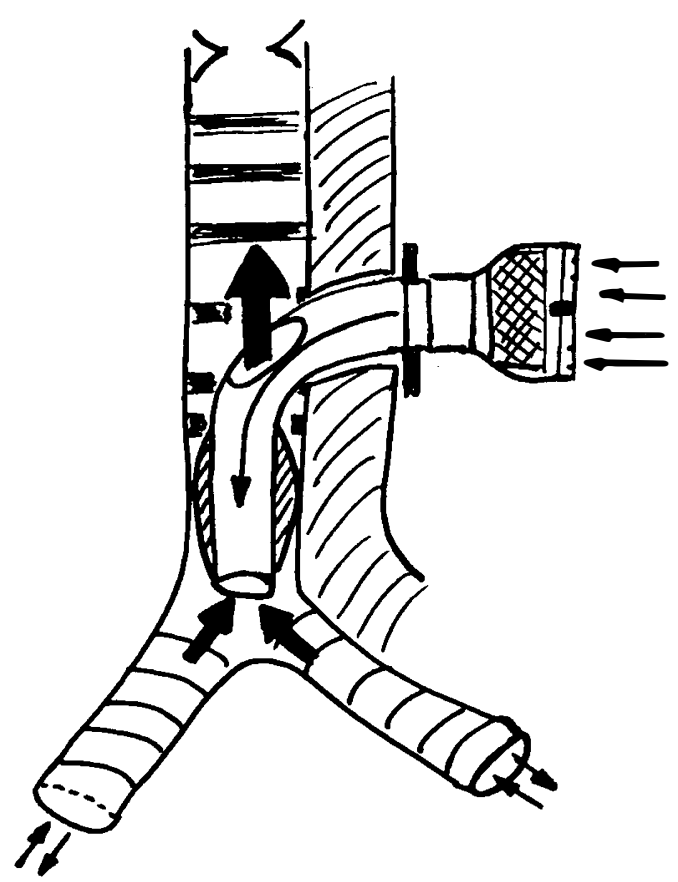

Figure 4 Ventilation with a cuffed fenestrated tube. 
effective. The carers attending the patient at home are trained to change the tube and to clean the stoma and with this system the patient is freed from discomfort and the process of changing the tube is both simple and safe.

Fibreoptic tracheoscopy should be performed every third to sixth month, checking both the position of the fenestration and the tip of the tube. Sometimes it is necessary to correct the length of the tube or to make some adjustment to the fenestration. Regarding tracheostomy problems, patients always return to our unit for special stoma review or for definitive plastic surgery on the tracheostomy. 